\title{
Performance Evaluation and Benchmarking of Event-Based Systems
}

SPEC Distinguished Dissertation Award 2011 (Invited Abstract)

\author{
Kai Sachs \\ SAP AG, Germany \\ kai.sachs@sap.com \\ Thesis Supervisor: \\ Alejandro Buchmann, TU Darmstadt, Germany \\ buchmann@dvs.tu-darmstadt.de
}

Event-based systems (EBS) are increasingly used as underlying technology in many mission critical areas and largescale environments, such as environmental monitoring and location-based services [3]. Moreover, novel event-based applications are typically highly distributed and data intensive with stringent requirements for performance and scalability. Since their reliability is crucial for the whole IT infrastructure, a certain Quality-of-Service (QoS) level has to be ensured. The motivation for our work was to support the development and maintenance of EBS that meet their QoS requirements. Given that EBS differ from traditional software in fundamental aspects such as their underlying communications paradigm, specific solutions and concepts are needed. System architects and deployers need tools and methodologies, which allow them to evaluate and forecast system performance and behavior in certain situations to identify potential performance problems and bottlenecks. Common approaches are benchmarking and performance modeling. However, no general performance modeling methodologies focusing on EBS had been published. Furthermore, there was a lack of test harnesses and benchmarks using representative workloads for EBS. Consequently, we focused on the development of a performance modeling methodology of EBS as well as on approaches to benchmark them. We summarize now our main contributions and proposed approaches.

To comprehend our contributions, an understanding of the fundamental ideas of EBS is essential. Generally, EBS are software systems in which an observed event triggers a reaction. For more details and definitions of events and related concepts refer to [3]. We evaluated the variety of underlying technologies with a focus on distributed EBS and messageorientend middlewares (MOMs) and provided a survey of products and standards [7]. In our review of existing work, we identified a lack of benchmarks and performance modeling approaches for EBS [5]. To support a structural evaluation of benchmarks, we introduced five categories of requirements [9]: (i) Representativeness: the benchmark has to be based on a representative workload. (ii) Comprehensiveness: exercise all platform features typically used in applications. (iii) Focus: place the emphasis on the technology server and minimize the impact of other services, e.g.,

Copyright is held by the author/owner(s).

ICPE'12, April 22-25, 2012, Boston, Massachusetts, USA

ACM 978-1-4503-1202-8/12/04. databases. (iv) Configurability: provide a configurable tool for performance analysis. (v) Scalability: provide ways to scale the workload in a flexible manner.

None of the existing benchmarks met all our requirements. Therefore, we saw a strong need for independent and standardized benchmarks for EBS fulfilling the requirements. To address this need we developed the first industry standard benchmark for EBS jointly with the SPEC (Standard Performance Evaluation Corporation). As underlying technology platform we chose Java Message Service (JMS) [12]. This was motivated by the fact that MOMs are widely used in industry and the quasi-standard for MOMs is JMS. Our efforts resulted in the SPECjms2007 standard benchmark [9]. Its main contributions were twofold: based on the feedback of industrial partners, we specified a comprehensive standardized workload with different scaling options and implemented the benchmark using a newly developed complex and flexible framework.

Using the SPECjms2007 benchmark we introduced a methodology for performance evaluation of message-oriented middleware platforms and showed how the workload can be tailored to evaluate selected performance aspects [10]. We demonstrated our methodology in a case study of a leading JMS platform and conducted in-depth performance analyses of the platform for a number of different workload and configuration scenarios.

The SPECjms2007 business scenario was specified independently from the underlying technology. Therefore, its usage is not limited to a specific type of EBS. We illustrated how the standardized workload can be applied to other EBS using the example of jms2009-PS, a benchmark for publish/subscribe-based communication [8]. This benchmark provides a flexible framework for performance analysis with a strong focus on research. The proposed benchmarks are now the de facto standard benchmarks for evaluating messaging platforms and have already been used successfully by several industrial and research organizations as a basis for further research on performance analysis of EBS.

To the best of our knowledge, no work introducing a general methodology for modeling EBS had been published. As a consequence, we investigated whether and how traditional performance modeling approaches are suitable to model the specifica of EBS. We introduced a formal definition of EBS and their performance aspects, which allows us, e.g., to describe workload properties and routing behavior in a structured way [6]. Resulting from our analysis of existing mod- 
eling techniques, we proposed an approach to characterize the workload and to model the performance aspects of EBS. We used operational analysis techniques to describe the system traffic and derived an approximation for the mean event delivery latency. We then showed how more detailed performance models based on queueing Petri nets (QPNs) [1] could be built and used to provide more accurate performance prediction. We chose QPNs as performance modeling technique because of their modeling power and expressiveness. Our approach allows evaluating and predicting the performance of an EBS and provides detailed system models. It can be used for an in-depth performance analysis and to identify potential bottlenecks. A further contribution is a terminology for performance modeling patterns targeting common aspects of event-based applications using QPNs [7, 11].

To additionally improve the modeling power of QPNs, we suggested several extensions of the standard QPNs, which allow building models in a more flexible and general way and address several limitations of QPNs [7]. By introducing a level of abstraction, it is possible to distinguish between logical and physical layers in our models. This enables to flexibly map logical to physical resources and thus makes it easy to customize the model to a specific deployment. The different layers allow reusing one logical model in several physical models or to map several logical models to one physical model. Furthermore, we addressed two limiting aspects of standard QPNs: constant cardinalities and the lack of transition priorities. By introducing non-constant cardinalities of transitions we increased the modeling flexibility and minimized the number of transition modes. The missing support of transition priorities in standard QPNs limits the control of the transition firing order. We addressed this restriction by incorporating priorities for transitions into QPNs and discussed several ways to implement them. Our extensions were integrated in the QPME / SimQPN software tools [4] or are planned for the upcoming release.

Finally, we validated the approach in two case studies. We applied our methodology to model EBS and predicted their performance and system behavior under load successfully. As part of the first case study we extended SIENA, a well-known distributed EBS [2], with a runtime measurement framework and predicted the runtime behavior including delivery latency for a basic workload [6] with a single event type. In the second case study, we developed a comprehensive model of the complete SPECjms2007 workload including the persistent layer, point-to-point and publish/subscribe communication [11]. To model the workload we applied our performance modeling patterns as well as our proposed QPN extensions.

We evaluated its accuracy in a commercial middleware environment. To validate our modeling technique we investigated deployments of the benchmark in representative environments comparing the model predictions against measurements on the real systems. A number of different scenarios with varying workload intensity (up to 30,000 messages / 4,500 transaction per second) and interaction mixes were taken into account. By means of the proposed models we were able to predict the performance accurately. No models of realistic systems of the size and complexity of the one considered in this case study exist in the literature.

Both case studies demonstrated the effectiveness and practicality of the proposed modeling and prediction methodology in the context of a real-world scenario. The advan- tage of the approach is that it is both practical and general, and it can be readily applied for performance evaluation of distributed EBS and MOM. The technique can be exploited as a powerful tool for performance prediction and capacity planning during the software engineering lifecycle of message-oriented event-driven systems.

Our results open up new avenues of research in the area of event-based systems. Our performance modeling methodology can be used to build self-adaptive EBS using automatic model extraction techniques. Such systems could dynamically adjust their configuration to ensure that QoS requirements are continuously met.

\section{REFERENCES}

[1] F. Bause. QN + PN = QPN - Combining Queueing Networks and Petri Nets. Tech. report no.461, Dept. of CS, University of Dortmund, Germany, 1993.

[2] A. Carzaniga, D. S. Rosenblum, and A. L. Wolf. Design and Evaluation of a Wide-Area Event Notification Service. ACM Trans. Comput. Syst., 19(3):332-383, 2001.

[3] A. Hinze, K. Sachs, and A. Buchmann. Event-based applications and enabling technologies. In Proc. of the ACM DEBS, 2009.

[4] S. Kounev and C. Dutz. QPME - A Performance Modeling Tool Based on Queueing Petri Nets. ACM SIGMETRICS PER, 36(4):46-51, 2009.

[5] S. Kounev and K. Sachs. Benchmarking and performance modeling of event-based systems. it Information Technology, 51(5):262-269, 2009.

[6] S. Kounev, K. Sachs, J. Bacon, and A. P. Buchmann. A methodology for performance modeling of distributed Event-Based systems. In Proc. of the IEEE ISORC, 2008.

[7] K. Sachs. Performance Modeling and Benchmarking of Event-Based Systems. PhD thesis, TU Darmstadt, 2010.

[8] K. Sachs, S. Appel, S. Kounev, and A. Buchmann. Benchmarking publish/subscribe-based messaging systems. In DASFAA 2010 - Int. Workshops: BenchmarX'10, LNCS. Springer, 2010.

[9] K. Sachs, S. Kounev, J. Bacon, and A. Buchmann. Workload characterization of the SPECjms2007 benchmark. In Proc. of the EPEW, LNCS. Springer, 2007.

[10] K. Sachs, S. Kounev, J. Bacon, and A. Buchmann. Performance evaluation of message-oriented middleware using the SPECjms2007 benchmark. Performance Evaluation, 66(8):410-434, 2009.

[11] K. Sachs, S. Kounev, and A. Buchmann. Performance modeling and analysis of message-oriented event-driven systems. Software and Systems Modeling, 2012. DOI: $10.1007 / \mathrm{s} 10270-012-0228-1$.

[12] Sun Microsystems, Inc. Java Message Service (JMS) Specification - Version 1.1. Technical report, 2002. 